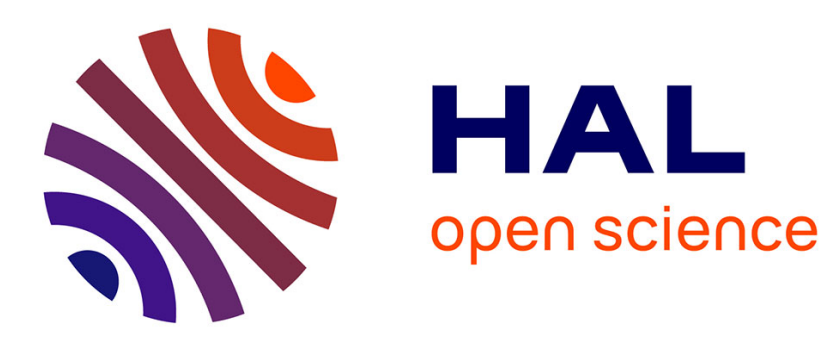

\title{
Consequences of self-preservation on the axis of a turbulent round jet
}

F. Thiesset, R. a. Antonia, L. Djenidi

\section{To cite this version:}

F. Thiesset, R. a. Antonia, L. Djenidi. Consequences of self-preservation on the axis of a turbulent round jet. Journal of Fluid Mechanics, 2014, 748, pp.R2. 10.1017/jfm.2014.235 . hal-01660265

\section{HAL Id: hal-01660265 \\ https://hal.science/hal-01660265}

Submitted on 28 Mar 2019

HAL is a multi-disciplinary open access archive for the deposit and dissemination of scientific research documents, whether they are published or not. The documents may come from teaching and research institutions in France or abroad, or from public or private research centers.
L'archive ouverte pluridisciplinaire HAL, est destinée au dépôt et à la diffusion de documents scientifiques de niveau recherche, publiés ou non, émanant des établissements d'enseignement et de recherche français ou étrangers, des laboratoires publics ou privés. 


\title{
Consequences of self-preservation on the axis of a turbulent round jet
}

\author{
F. THIESSET ${ }^{1}$, R. A. A N T ONIA ${ }^{1}$, \\ AND L. D JENID I ${ }^{1} \dagger$ \\ ${ }^{1}$ School of Engineering, University of Newcastle, Callaghan Campus, NSW 2308, Australia
}

(Received November 17, 2016)

On the basis of a two-point similarity analysis, the well-known power law variations for the mean kinetic energy dissipation rate $\bar{\epsilon}$ and the longitudinal velocity variance $\overline{u^{2}}$ on the axis of a round jet are derived. In particular, the prefactor for $\bar{\epsilon} \propto\left(x-x_{0}\right)^{-4}$, where $x_{0}$ is a virtual origin, follows immediately from the variation of the mean velocity, the constancy of the local turbulent intensity and the ratio between the axial and transverse velocity variance.

Second, the limit at small separations of the two-point budget equation, yields an exact relation illustrating the equilibrium between the skewness of longitudinal velocity derivative $S$ and the destruction coefficient $G$ of enstrophy. By comparing the latter relation with that for homogeneous isotropic decaying turbulence, it is shown that the approach towards the asymptotic state at infinite Reynolds number of $S+2 G / R_{\lambda}$ in the jet differs from that in purely decaying turbulence, although $S+2 G / R_{\lambda} \propto R_{\lambda}^{-1}$ in each case. This suggests that, at finite Reynolds numbers, the transport equation for $\bar{\epsilon}$ imposes a fundamental constraint on the balance between $S$ and $G$ which depends on the type of large-scale forcing and may thus differ from flow to flow. This questions the conjecture that $S$ and $G$ follow a universal evolution with $R_{\lambda}$; instead, $S$ and $G$ must be tested separately in each flow. The implication for the constant $C_{\epsilon 2}$ in the $k-\bar{\epsilon}$ model is also discussed.

\section{Introduction}

The turbulent round jet is an important canonical decaying shear flow and its study remains valuable in the context of fundamental turbulence research. Also, it features in many practical applications, from pollutant dispersion to the design of efficient chemical reactors (e.g. Baldyga et al. (1994)). The hypothesis of self-preservation, which assumes that the flow is governed by a single set of length and velocity scales, has been extensively used for describing the spatial evolution of one-point statistics in the far field of a round jet (e.g. Panchapakesan \& Lumley (1993)). Extending the analysis of self-preservation to two-point statistics provides a deeper insight into the flow details since the evolution of the turbulence structure at a given scale can be assessed. Burattini et al. (2005b,a) were the first to consider such an analysis on the axis of a round jet using structure functions and spectra. Ewing et al. (2007) further extended the similarity analysis to the entire flow using correlation functions. These studies suggested that self-similar solutions for the terms in the two-point budget equation exist, independently of the Reynolds number. Perhaps more importantly, Burattini et al. (2005b) suggested that since the Reynolds number $R_{\lambda}=u^{\prime} \lambda / \nu$ ( $\lambda$ is the Taylor microscale, to be defined later and $u^{\prime}$ a typical

$\dagger$ Email address for correspondence: Lyazid.Djenidi@newcastle.edu.au 
velocity fluctuation) is constant during the decay, the round jet flow may assume selfsimilar solutions, independently of the choice of similarity variables that can be made. This particularity of the round jet flow considerably simplifies the similarity analysis since inner scales (e.g. the Taylor microscale $\lambda$, the integral length scale $L_{u}$ or the Kolmogorov length-scale $\eta$, altogether defined later in the manuscript) can be used interchangeably with outer scales (the half-width for instance) because they all behave similarly during the decay. This circumvents the question of which are the most relevant similarity variables to be used for normalization. With the exception of the (very) far field of a plane wake for which the constancy of the Taylor microscale Reynolds number is likely to be attained only asymptotically, and the initial period of decay of grid turbulence at infinitely large Reynolds number, the round jet is the only decaying flow for which $R_{\lambda}=$ constant.

One important consequence of self-similarity in the far field of a round jet is that single-point turbulent quantities should behave in a power-law fashion. For instance, the inner and outer length-scales are proportional to the streamwise distance $x$, the characteristic velocities follow an hyperbolic decay rate proportional to $\left(x-x_{0}\right)^{-1}$ and the mean kinetic energy dissipation rate $\bar{\epsilon}$ varies as $\left(x-x_{0}\right)^{-4}\left(x_{0}\right.$ is a virtual origin). The latter relation is generally derived by assuming that the normalized dissipation rate parameter $C_{\epsilon}=\bar{\epsilon} L_{u} / u^{\prime 3}$ is constant during the decay (Antonia et al. 1980). It will be shown in $\S 2$ of this paper that this hypothesis is not required to obtain the -4 power-law for $\bar{\epsilon}$.

Since the pioneering work of Kolmogorov (1941), the mean dissipation rate is often considered as one of the most critical quantities in the study of turbulence. However, the transport equation for $\bar{\epsilon}$ has generally been given much less attention by comparison to e.g. the one-point kinetic energy budget. This is surprising considering that this equation is involved in the majority of closure models, e.g. the $k-\bar{\epsilon}$ model. A reliable parametrization of this type of closure equation requires a basic understanding of the mechanisms underpinning the evolution of $\bar{\epsilon}$ and its relationships to the large-scales. A particular motivation of the present study is to provide, within this perspective, some insight into the transport equation of $\bar{\epsilon}$ along the axis of a round jet.

To this end, we extend the analysis of Burattini et al. $(2005 b, a)$ in a significant manner by examining in detail some consequences of self-preservation for the two-point energy budget equation on the axis of a round jet. In section $\S 2$, it is first shown that the powerlaw variations for $\bar{\epsilon}$, the longitudinal velocity variance $\overline{u^{2}}$ and all subsequent quantities can be derived from the constraint imposed by self-preservation of the two-point transport equation. Attention is also paid to the prefactor for $\bar{\epsilon} \propto\left(x-x_{0}\right)^{-4}$. Then, the limit at small separation of the scale-by-scale budget is applied in $\S 3$ and yields the transport equation of the enstrophy (or equivalently that of $\bar{\epsilon}$ if local homogeneity holds). More precisely, a relation highlighting the equilibrium between the production $S$ and destruction $G$ of enstrophy (or dissipation rate) is obtained. This relation is compared to that of homogeneous decaying turbulence and implications for the $k-\bar{\epsilon}$ model are discussed.

\section{Self-preservation on the axis of a round jet}

On the axis of an axisymmetric jet flow, the experimental investigation of Burattini et al. (2005b) suggested that the energy budget equation is given by

$$
-\frac{3}{r^{4}} \int_{0}^{r} s^{4}\left[\bar{U} \frac{\partial \overline{(\Delta u)^{2}}}{\partial x}+2\left(\overline{(\Delta u)^{2}}-\overline{(\Delta v)^{2}}\right) \frac{\partial \bar{U}}{\partial x}\right] d s-\overline{(\Delta u)^{3}}+6 \nu \frac{\partial}{\partial r} \overline{(\Delta u)^{2}}=\frac{4}{5} \bar{\epsilon} r
$$


Eq. (2.1) may be valid over a range of scales over which local isotropy is satisfied. $u$ and $v$ correspond to the fluctuating velocity component in the longitudinal $x$ and transverse direction $y$ respectively. $\nu$ is the kinematic viscosity and $\bar{\epsilon}=15 \nu \overline{(\partial u / \partial x)^{2}}$ stands for the mean energy dissipation rate. The overbar denotes averaging and $s$ is a dummy integration variable. $\Delta u=u(x+r)-u(x)$ is the velocity increment between two points separated by a distance $r$. Eq. (2.1) is the generalization of the Kolmogorov (1941) equation which accounts for the forcing acting at rather large scales. The first term corresponds to the decay of second-order structure function in the streamwise direction $x$ whilst the second term underlines the effect of production through the longitudinal mean velocity gradient. In the context of infinite Reynolds numbers, and for scales sufficiently small, these terms can be neglected and Eq. (2.1) reduces to the Kolmogorov (1941) equation.

Applying the limit at large separations to Eq. (2.1), yields

$$
-\frac{1}{2} \bar{U} \frac{\partial \overline{q^{2}}}{\partial x}-\left(\overline{u^{2}}-\overline{v^{2}}\right) \frac{\partial \bar{U}}{\partial x}=\bar{\epsilon}
$$

where $\overline{q^{2}}=\overline{u^{2}}+2 \overline{v^{2}}$ by virtue of axisymmetry. Eq. (2.2) is the one-point kinetic energy budget and indicates that the mean energy dissipation rate compensates both advection and production effects. The pressure-transport term has been neglected in Eq. (2.2) as was done in Burattini et al. (2005a); Panchapakesan \& Lumley (1993); Taub et al. (2013); the DNS data of Taub et al. (2013) provide some support for this on the jet axis. For the data of Burattini et al. (2005a), the contribution from the production term is about one tenth of that from the advection. Lipari \& Stansby (2011) have reviewed the differences between previously published one-point kinetic energy budgets and confirm this.

For the range of scales over which Eq. (2.1) is valid, let us suppose that second- and third-order structure functions can be written as

$$
\begin{aligned}
\overline{(\Delta u)^{2}}(x, r) & =u_{K}^{2}(x) f^{*}\left(r^{*}\right) \\
\overline{(\Delta v)^{2}}(x, r) & =u_{K}^{2}(x) h^{*}\left(r^{*}\right) \\
-\overline{(\Delta u)^{3}}(x, r) & =u_{K}^{3}(x) g^{*}\left(r^{*}\right),
\end{aligned}
$$

where $u_{K}=(\nu \bar{\epsilon})^{1 / 4}$ is the Kolmogorov characteristic velocity and $r^{*}=r / \eta$ with $\eta=$ $\left(\nu^{3} / \bar{\epsilon}\right)^{1 / 4}$ the Kolmogorov length-scale. In Burattini et al. $(2005 b)$, it was revealed that equilibrium similarity for the scale-by-scale budget yields the Taylor microscale as the relevant normalization length-scale. However, as emphasized by Burattini et al. (2005b), the constancy of $R_{\lambda}$ suggests that the degree with which structure functions and spectra comply with similarity should be equally satisfactory, regardless of the choice of scaling parameters, and complete self-similarity may be reached. In other words, Kolmogorov scaling can be used interchangeably with George (1992) similarity variables $(\equiv \lambda$ and $\overline{u^{2}}$ ) or integral scales ( $\equiv L_{u}$ and $\overline{u^{2}}$ ). Use was made here of the Kolmogorov similarity variables, hereafter indicated by an asterisk. The scale-by-scale budget Eq. (2.1) can thus be written as

$$
\left[\frac{3 \bar{U}}{u_{K}} \frac{d \eta}{d x}\right] \frac{\Gamma_{1}^{*}}{r^{* 4}}-\left[\frac{3 \bar{U}}{\bar{\epsilon}} \frac{d u_{K}^{2}}{d x}\right] \frac{\Gamma_{2}^{*}}{r^{* 4}}-\left[\frac{6 u_{K}^{2}}{\bar{\epsilon}} \frac{\partial \bar{U}}{\partial x}\right] \frac{\Gamma_{2}^{*}-\Gamma_{3}^{*}}{r^{* 4}}+[1] g^{*}+[6] f^{* \prime}=\left[\frac{4}{5}\right] r^{*}
$$

where $\Gamma_{1}^{*}=\int_{0}^{r^{*}} s^{* 5} f^{* \prime} d s^{*}, \Gamma_{2}^{*}=\int_{0}^{r^{*}} s^{* 4} f^{*} d s^{*}, \Gamma_{3}^{*}=\int_{0}^{r^{*}} s^{* 4} h^{*} d s^{*}$, and the prime denotes differentiation with respect to $r^{*}$. Complete self-preservation requires that all terms within brackets behave similarly with $x$. Since three appear to be constant, this implies 
the following conditions for the other three

$$
\begin{aligned}
\frac{3 \bar{U}}{u_{K}} \frac{d \eta}{d x} & =C_{1} \\
-\frac{3 \bar{U}}{\bar{\epsilon}} \frac{d u_{K}^{2}}{d x} & =C_{2} \\
-\frac{6 u_{K}^{2}}{\bar{\epsilon}} \frac{\partial \bar{U}}{\partial x} & =C_{3},
\end{aligned}
$$

where the constants $C_{1}, C_{2}$ and $C_{3}$ need to be evaluated. As discussed in Burattini et al. (2005b) and Ewing et al. (2007), conservation of mean momentum requires the longitudinal mean velocity $\bar{U}$ to behave as follows

$$
\frac{\bar{U}}{U_{j}}=A_{U}\left(\frac{x-x_{0}}{D}\right)^{-1} .
$$

where $U_{j}$ is the jet exit velocity, $D$ is the nozzle diameter and $A_{U}$ is the power-law prefactor for $\bar{U}$. Combining Eqs. (2.5a) and (2.6) leads to

$$
\frac{\bar{\epsilon} D}{U_{j}^{3}}=\left[\frac{3 A_{U}}{C_{1} R e_{D}^{1 / 2}}\right]^{2}\left(\frac{x-x_{0}}{D}\right)^{-4}
$$

where $R e_{D}=U_{j} D / \nu$. Eq. (2.7) emphasizes that the well-known power-law evolution

$$
\frac{\bar{\epsilon} D}{U_{j}^{3}}=A_{\epsilon}\left(\frac{x-x_{0}}{D}\right)^{-4}
$$

is recovered. $A_{\epsilon}$ is the power-law prefactor for $\bar{\epsilon}$ and will be evaluated further. Using Eqs. $(2.5 b)$ and $(2.5 c)$, it can be shown that $C_{3}=C_{2}=2 C_{1}$.

In order to assess the evolution of the longitudinal velocity variance, the one-point energy budget of turbulent kinetic energy Eq. (2.2) is used. Injecting Eqs. (2.6) and (2.8) into Eq. (2.2), yields a non-linear first-order differential equation of the form

$$
\mathcal{A} \hat{x} \frac{\partial \mathcal{F}}{\partial \hat{x}}+\mathcal{B F}=\mathcal{C} \hat{x}^{-2}
$$

where $\hat{x}=\left(x-x_{0}\right) / D, \mathcal{F}=\overline{u^{2}} / U_{j}^{2}, \mathcal{A}=-1 / 2-\mathcal{R}, \mathcal{B}=1-\mathcal{R}, \mathcal{C}=A_{\epsilon} / A_{U}, \mathcal{R}=\overline{v^{2}} / \overline{u^{2}}$. Seeking a solution for $\mathcal{F}$ of the form $\mathcal{F}=\mathcal{D} \hat{x}^{\alpha}$, we finally obtain

$$
\frac{\overline{u^{2}}}{U_{j}^{2}}=\left[\frac{A_{\epsilon}}{(2+\mathcal{R}) A_{U}}\right]\left(\frac{x-x_{0}}{D}\right)^{-2} .
$$

Therefore, the power-law decay for the longitudinal velocity variance is also deduced. In addition, the local turbulence intensity

$$
A_{I}=\frac{\sqrt{\overline{u^{2}}}}{\bar{U}}=\left[\frac{A_{\epsilon}}{(2+\mathcal{R}) A_{U}^{3}}\right]^{1 / 2}
$$

is constant with respect to $x$ provided $\mathcal{R}$ does not vary with $x$ (self-preservation for onepoint statistics requires $\mathcal{R}$ to be constant). We recall here that a reliable estimation of the mean energy dissipation rate is a challenging task for the experimentalist. Eq. (2.11) provides a simple means for estimating $\bar{\epsilon}$ once $\bar{U}, \overline{u^{2}}$ and $\mathcal{R}$ are known, viz.

$$
\frac{\bar{\epsilon} D}{U_{j}^{3}}=\left[(2+\mathcal{R}) A_{U}^{3} A_{I}^{2}\right]\left(\frac{x-x_{0}}{D}\right)^{-4} .
$$


TABLE 1. Experimental and predicted values for the prefactors $A_{\epsilon}$ and $A_{R_{\lambda}}$ provided $\mathcal{R}=2 / 3$ as in Burattini et al. (2005b) and the respective experimental values for $A_{U}$ and $A_{I}$. Experimental data are taken from Mi et al. (2013) (MXZ), Friehe et al. (1972) (FVAG) and Burattini et al. $(2005 b)(\mathrm{BAD})$

MXZ

\begin{tabular}{lcccccccccc}
$R e_{D}\left(10^{3}\right)$ & 4.05 & 5.40 & 6.75 & 8.05 & 10.75 & 13.5 & 16.35 & 20.10 & 120 & 113 \\
& & & & & & & & & & \\
$A_{\epsilon}$ & 18.5 & 28.7 & 32.0 & 41.7 & 47.5 & 49.2 & 50.7 & 49.9 & 48 & 42.5 \\
Eq. $(2.12)$ & 21.5 & 27.5 & 28.9 & 32.3 & 34.1 & 35.5 & 36.0 & 35.4 & 39.7 & 32.7 \\
Error (\%) & 16 & 4 & 10 & 29 & 39 & 38 & 41 & 41 & 21 & 30 \\
& & & & & & & & & & \\
$A_{R_{\lambda}}$ & & & & & 1.16 & & & & 1.56 & 1.33 \\
Eq. $(2.15)$ & 1.23 & 1.25 & 1.23 & 1.26 & 1.28 & 1.29 & 1.30 & 1.29 & 1.39 & 1.26 \\
Error (\%) & 6 & 8 & 6 & 9 & 10 & 12 & 12 & 11 & 12 & 5 \\
\hline
\end{tabular}

Even though assuming $C_{\epsilon}=$ constant (which ensues from the hypothesis of self-preservation on the jet axis) also leads to the -4 power-law for $\bar{\epsilon}$, it is worth noting that the magnitude of $C_{\epsilon}$ is not needed here for the prefactor $A_{\epsilon}$ to be derived. It follows that the Kolmogorov characteristic scales should behave as

$$
\begin{gathered}
\frac{\eta}{D}=\left[(2+\mathcal{R}) R e_{D}^{3} A_{U}^{3} A_{I}^{2}\right]^{-1 / 4}\left(\frac{x-x_{0}}{D}\right), \\
\frac{u_{K}}{U_{j}}=\left[\frac{(2+\mathcal{R}) A_{U}^{3} A_{I}^{2}}{\operatorname{Re}_{D}}\right]^{1 / 4}\left(\frac{x-x_{0}}{D}\right)^{-1},
\end{gathered}
$$

and therefore

$$
\overline{u^{* 2}}=\frac{\overline{u^{2}}}{u_{K}^{2}}=\left[\frac{A_{U} A_{I}^{2} R e_{D}}{2+\mathcal{R}}\right]^{1 / 2}
$$

is constant, independently of $x$. Since $\overline{u^{* 2}}=R_{\lambda} / \sqrt{15}$, where $R_{\lambda}=\left(\overline{u^{2}}\right)^{1 / 2} \lambda / \nu$ is the Reynolds number based on the Taylor microscale $\lambda=\left(15 \nu \overline{u^{2}} / \bar{\epsilon}\right)^{1 / 2}$, it follows that

$$
A_{R_{\lambda}}=\frac{R_{\lambda}}{R e_{D}^{1 / 2}}=\left[\frac{15 A_{U} A_{I}^{2}}{2+\mathcal{R}}\right]^{1 / 2} .
$$

It is thus not surprising to observe that complete self-preservation requires $R_{\lambda}$ to be constant during the decay. The definition of the Taylor microscale leads to

$$
\frac{\lambda}{D}=\left[\frac{15}{(2+\mathcal{R}) R e_{D} A_{U}}\right]^{1 / 2}\left(\frac{x-x_{0}}{D}\right)
$$

Since the constant $C_{1}$ is related to $A_{\epsilon}$ via Eq. (2.7), the final equation for $f^{*}$ on the axis of turbulent round jet can be written as

$$
\left(\frac{3 \sqrt{15}}{2+\mathcal{R}}\right) R_{\lambda}^{-1}\left(\Gamma_{1}^{*}+4 \Gamma_{2}^{*}-2 \Gamma_{3}^{*}\right) r^{*-4}+g^{*}+6 f^{\prime *}=\frac{4}{5} r^{*}
$$

Note that Eq. (2.17) explicitly indicates that the large-scale terms should vanish when $R_{\lambda} \rightarrow \infty$.

In Table 1, a sample a different experimental values for $A_{\epsilon}$ and $A_{R_{\lambda}}$ are compared with values inferred from Eqs. (2.12) and (2.15) respectively, with $\mathcal{R}=2 / 3$ (Burattini et al. 
$2005 b)$ and the respective experimental values for $A_{U}$ and $A_{I}$. Predictions are generally in satisfactory agreement with experiments, although lower on average by about $25 \%$ for $A_{\epsilon}$ whereas discrepancies for $A_{R_{\lambda}}$ are on average of about $10 \%$.

The systematic difference between experimental and predicted values for $A_{\epsilon}$ leads us to question the validity of Eq. (2.1). Indeed, this suggests that the turbulent diffusion and/or pressure transport terms may contribute to the scale-by-scale budget Eq. (2.1) and consequently affect the value of $A_{\epsilon}$. In addition, apart from the difficulty of measuring $\bar{\epsilon}$ (see for example the detailed review of hot wire measurements of small scale quantities by Antonia et al. (1986)) that may also explain the discrepancy for $A_{\epsilon}$, the inadequate estimation of the virtual origin is likely to have a significant effect. Indeed, since the decay exponent for $\bar{\epsilon}$ is -4 , small variations of $x_{0}$ significantly alter the value of the prefactor. A more precise estimation of $x_{0}$ could be handled by plotting $\bar{\epsilon} D / U_{j}^{3}\left(x-x_{0} / D\right)^{4}$ for different values of $x_{0}$ and then identifying the value which yields the best plateau. Finally, the value of $\mathcal{R}$ which was not measured by Mi et al. (2013) and Friehe et al. (1972) may differ between different experiments depending on initial/boundary conditions. More generally, it is not out of the question that different initial/boundary conditions may affect the make up of the one-point kinetic energy budget.

It seems clear that the difference between the predicted and measured values of $A_{\epsilon}$ are due to a large extent to an error in $x_{0}$ since discrepancies for $A_{R_{\lambda}}$ are much less important, the latter being independent of the estimation of the virtual origin. For the data of Mi et al. (2013), the range of $x(20 D \leq x \leq 30 D)$ is also likely to be too limited for a reliable fit to be applied. Further, self-similarity is questionable over this range.

In summary, providing that both local isotropy and complete self-similarity hold, and assuming that only the production and advection terms have to be retained for Eq. (2.1) to be valid on the axis of a round jet, the similarity analysis provided here leads to an exact expression for $\bar{\epsilon}, \overline{u^{2}}$ and all subsequent quantities. Further insight is provided into the relationship between the prefactors $A_{U}, A_{I}, A_{\epsilon}$ and $A_{R_{\lambda}}$. We show that a knowledge of only $A_{U}, A_{I}$ and $\mathcal{R}$ may be sufficient for $A_{\epsilon}$ and $A_{R_{\lambda}}$ to be assessed indirectly. Experimental data provide satisfactory support for this. Note also that this analysis indicates that the virtual origin $x_{0}$ should be same, i.e. independent of the statistical quantity under consideration. This contrasts with the observation of Mi et al. (2013) which revealed substantial differences in the value of $x_{0}$ depending on the quantity used to assess it.

\section{Consequences for the mean energy dissipation rate transport equation}

When $r \rightarrow 0$, Eq. (2.1) reduces to the one-point transport equation for $\bar{\epsilon}$ (Antonia et al. (2000)). This follows by first writing a Taylor series expansion for $\overline{(\Delta u)^{2}}$ and $\overline{(\Delta v)^{2}}$

$$
\overline{(\Delta \alpha)^{2}}=\overline{\left(\frac{\partial \alpha}{\partial x}\right)^{2}} r^{2}-\frac{1}{12} \overline{\left(\frac{\partial^{2} \alpha}{\partial x^{2}}\right)^{2}} r^{4}+\mathcal{O}\left(r^{6}\right),
$$

where $\alpha$ stands for either $u$ or $v$. Local isotropy requires $\overline{(\partial u / \partial x)^{2}}=\bar{\epsilon} / 15 \nu$ and $\overline{(\partial v / \partial x)^{2}}=$ $2 \bar{\epsilon} / 15 \nu$. After equating terms in $r^{3}$ (see Antonia et al. (2000)), the final transport equation for $\bar{\epsilon}$ is

$$
-\bar{U} \frac{\partial \bar{\epsilon}}{\partial x}+2 \frac{\partial \bar{U}}{\partial x} \bar{\epsilon}=\frac{7}{3 \sqrt{15}} \frac{\bar{\epsilon}^{3 / 2}}{\nu^{1 / 2}}\left[S+2 \frac{G}{R_{\lambda}}\right]
$$




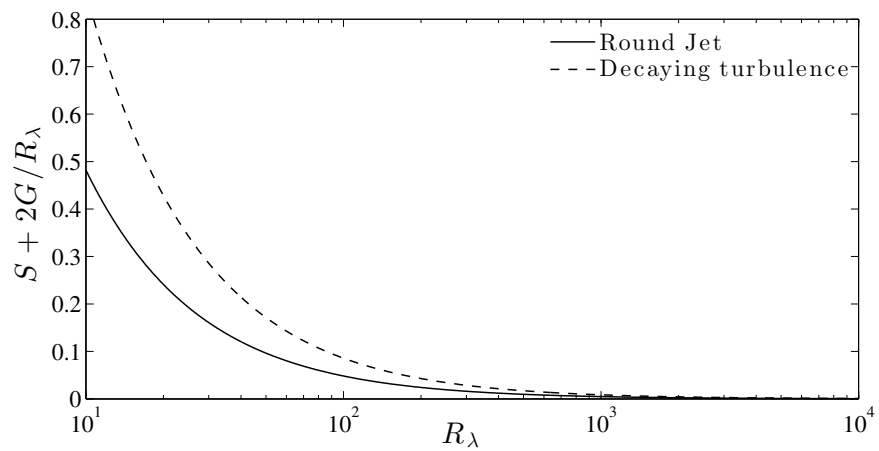

FIgURE $1 . S+2 G / R_{\lambda}$ as a function of $R_{\lambda}$ in decaying (dashed line) and on the axis of a round jet (solid line). For the purely decaying flow, $\mathcal{R}=1$ and $n_{u}=1$ whereas for the jet flow $\mathcal{R}=2 / 3$.

with

$$
S=\frac{\overline{(\partial u / \partial x)^{3}}}{\left[\overline{(\partial u / \partial x)^{2}}\right]^{3 / 2}} \quad \text { and } \quad G=\frac{\overline{u^{2}} \overline{\left(\partial^{2} u / \partial x^{2}\right)^{2}}}{\left[\overline{(\partial u / \partial x)^{2}}\right]^{2}}
$$

If similarity applies, $\bar{U}$ behaves as per Eq. (2.6) while $S, G$ and $R_{\lambda}$, cannot vary with $x$. The magnitudes of these quantities can however depend on the initial conditions. If $\bar{\epsilon} \sim\left(x-x_{0}\right)^{-n}$, then $n$ can only equal 4 for Eq. (3.2) to be satisfied. This is consistent with our earlier deduction from the two-point energy budget.

Injecting Eqs. (2.6) and (2.8) into Eq. (3.2) and further using Eqs. (2.12) and (2.15), one obtains

$$
S+\frac{2 G}{R_{\lambda}}=\frac{90}{7(2+\mathcal{R})} R_{\lambda}^{-1}
$$

which is an exact relation. The main objective in deriving Eq. (3.4) is to assess the way $S+2 G / R_{\lambda}$ approaches zero along the jet axis as $R_{\lambda}$ increases indefinitely and compare with decaying homogeneous isotropic turbulence. In the latter case, $S+2 G / R_{\lambda}$ is given by (Batchelor \& Townsend 1947; Lee et al. 2013)

$$
S+\frac{2 G}{R_{\lambda}}=\frac{90}{7(1+2 \mathcal{R})}\left(\frac{1+n_{u}}{n_{u}}\right) R_{\lambda}^{-1},
$$

where $n_{u}>0$ is the decay exponent of the longitudinal velocity variance. These two exact equations (Eqs. (3.4) and (3.5)) reveal that $S+2 G / R_{\lambda}$ will reach an asymptotic state at an infinite Reynolds number corresponding to (locally) stationary turbulence, i.e. $S+2 G / R_{\lambda}=0$. For finite Reynolds number flows, the equilibrium between $S$ and $G$, two (very) small scale quantities, is likely to be altered by initial conditions (a large-scale phenomenon), through the large-scale anisotropy ratio $\mathcal{R}$ and the decay exponent $n_{u}$ for the purely decaying case. Note that $S+2 G / R_{\lambda}$ in each case approaches zero at the same rate, i.e. $S+2 G / R_{\lambda} \propto R_{\lambda}^{-1}$. In addition, one important comment is that even though internal intermittency may possibly influence the respective magnitudes of $S$ (Van Atta \& Antonia 1980; Sreenivasan \& Antonia 1997) and $G$, the equilibrium between $S$ and $G$ as given by Eq. (3.4) or (3.5) cannot be altered by such effects.

The approach of $S+2 G / R_{\lambda}$ towards the asymptotic value of zero is represented in Fig. 1. For the purely decaying case, use was made of $\mathcal{R}=1$ and $n_{u}=1$, the latter value leading to complete self-preservation since it implies $R_{\lambda}=$ constant. The reader is referred to Lee et al. (2013) who discussed the effect of varying $n_{u}$ on $S+2 G / R_{\lambda}$. For the 
jet flow, a value of $\mathcal{R}=2 / 3$ is adopted on the basis of data by Burattini et al. $(2005 b)$. It is clear that the two flows will reach the asymptotic state following two distinct routes, albeit in the same hyperbolic fashion, viz. $S+2 G / R_{\lambda} \propto R_{\lambda}^{-1}$. On the jet axis $S+2 G / R_{\lambda}$ is always closer to zero by comparison to the purely decaying case. This difference is only attributed to some large-scale effects, namely (i) the large-scale anisotropy ratio, (ii) the decay exponent for the purely decaying case and (iii) more importantly through the additional effect of the production term in the scale-by-scale budget pertaining to the jet flow. Lindborg (1999); Lundgren (2002); Antonia \& Burattini (2006) demonstrated that the maximum value of the non-linear transfer term approaches the asymptote of $4 / 5$ with a scaling of the form $R_{\lambda}^{-2 / 3}$. It is thus interesting to note that inertial range scales loose the information about the large-scales at lower rate $R_{\lambda}^{-2 / 3}$ by comparison with the smallest scales, for which the scaling is $R_{\lambda}^{-1}$.

In summary, applying the limit at small separation of the scale-by-scale budget, the evolution of $\bar{\epsilon}$ with $x$ on the axis of a round jet is, not surprisingly, recovered in agreement with our previous deduction from the two-point similarity analysis. Further, the transport equation for the mean dissipation rate on the jet axis yields an exact equation for the balance between $S$ and $G$ as a function of the Reynolds number. This equation may be valid independently of $R_{\lambda}$, providing that local isotropy holds, that complete self preservation is attained and depends on the particular form assumed for the two-point budget equation. If only the production and advection terms are retained, $S+2 G / R_{\lambda}$ then appears to depend on the large-scales via the anisotropy ratio $\mathcal{R}$. The trend of $S+2 G / R_{\lambda}$ towards the asymptote is also compared to the one for a purely decaying flow where production is absent. For these two flows, the asymptotic state is reached along two different routes, although in each case $S+2 G / R_{\lambda} \propto R_{\lambda}^{-1}$.

Another important consequence of the present analysis relates to some modeling issues. As discussed by Pope (1978), it is generally found that the constants appearing in closure models such as the $k-\bar{\epsilon}$ model have to be adjusted depending on the flow under consideration. The analysis provided here may explain this anomaly. Indeed, let us start by rewriting Eq. (3.2) as

$$
-\bar{U} \frac{\partial \bar{\epsilon}}{\partial x}=\mathcal{P}+C_{\epsilon 2} \frac{\bar{\epsilon}^{2}}{k}
$$

which is generally referred to as the $\bar{\epsilon}$ equation in the $k-\bar{\epsilon}$ model where $k=\overline{q^{2}} / 2$ and $\mathcal{P}$ denotes a production term. It thus appears that $C_{\epsilon 2}$ is explicitly related to $S$ and $G$ via

$$
C_{\epsilon 2}=\frac{7}{90}(1+2 \mathcal{R})\left[S R_{\lambda}+2 G\right]
$$

Injecting either Eq. (3.4) or (3.5) into Eq. (3.7) leads to

$$
\begin{cases}C_{\epsilon 2}=\frac{n_{u}+1}{n_{u}} & \text { in decaying turbulence } \\ C_{\epsilon 2}=\frac{1+2 \mathcal{R}}{2+\mathcal{R}} & \text { on the axis of a round jet }\end{cases}
$$

Therefore, with $\mathcal{R}=2 / 3$, one then obtains $C_{\epsilon 2}=7 / 8$ in the jet flow which is very different from the value of $2\left(\equiv n_{u}=1\right)$ pertaining to a purely decaying turbulence. At this stage, it is clear that the value of $C_{\epsilon 2}$ in the $k-\bar{\epsilon}$ model will differ depending on the form Eq. (3.2) will assume in each flow. In addition, since the balance between the advection, production, diffusion and pressure transport terms varies from location to location in the flow, one should also expect similar variations for $C_{\epsilon 2}$. This confirms that any notion of universality for $C_{\epsilon 2}$ is untenable. 


\section{Conclusions}

All the results presented here rely essentially on three main hypotheses: local isotropy, complete self-preservation, and the particular terms retained in Eq. (2.1). The first hypothesis, which only needs to be valid at the smallest scales (the scale of velocity derivatives) for Eq. (3.4) to be valid, does not appear to be too restrictive. We recall that Antonia \& Mi (1993) showed that the departure from isotropy of $\bar{\epsilon}_{\theta}$, the temperature variance dissipation rate is smaller in the self-preserving region of a round jet than for other shear flows. Recent experiments by Darisse et al. (2014) confirm the small departure from isotropy for $\bar{\epsilon}_{\theta}$ on the jet axis. One expects that $\bar{\epsilon}$ should also exhibit a small departure from isotropy since the scalar field is usually more anisotropic than the velocity field. Even when departures from isotropy cannot be ignored, as for example on the axis of a plane jet, the locally isotropic form of the enstrophy equation appears to be satisfied (Antonia et al. 1986). In addition, instead of invoking the isotropic two-point budget equation as given by Eq. (2.1), all the present results may be inferred on the basis of the axisymmetric budget equation as provided by Danaila et al. (2012) with similar deductions. The assumption of complete self-preservation appears to depend only on the constancy of the Reynolds number during the decay. Here again, this assumption is well verified on the axis of a round jet. However, there remains some doubts as to whether the particular forms for the one-point and two-point kinetic energy budget are known with sufficient accuracy. As far as the axis of a round jet is concerned, Burattini et al. (2005b); Panchapakesan \& Lumley (1993); Taub et al. (2013) suggested that only the advection and production terms feature in Eq. (2.2). DNS data obtained at larger $x / D$ and $R e_{D}$ than in previous simulations could be desirable to confirm this.

One of the main outcomes of the present analysis is to underline the very broad potential offered by the scale-by-scale budget. Since the latter is derived directly from the Navier-Stokes equation, all the results can be considered as exact, provided that the three hypotheses mentioned above are respected. The power-law evolutions for $\bar{\epsilon}, \overline{u^{2}}$ and all subsequent quantities are not based on empirical observations but are derived analytically in an exact manner. The virtual origin is also proven to be the same independently of the quantity under consideration. Further, the analysis yields a very simple way of assessing the mean energy dissipation rate and the ratio between $R_{\lambda}$ and $R e_{D}$ provided $A_{U}, \mathcal{R}$ and $A_{I}$, i.e. three readily measurable quantities, are known.

A last important comment ensues from our results. Indeed, Eqs. (3.4) and (3.5) are exact relations and it was shown that the equilibrium between $S$ and $G$ cannot be altered by any intermittency effects. The latter relations thus represent an important constraint that must be considered by any phenomenological theory of small scale turbulence. They also highlight the dependence between large and small scales, in part through the appearance of the large-scale anisotropy ratio. Large and small scales will therefore be statistically uncorrelated only at infinite Reynolds numbers for which $S+2 G / R_{\lambda} \rightarrow 0$. In addition, it imposes a fundamental dynamical constraint on the way $S$ and $G$ behave with respect to $R_{\lambda}$. The different routes along which $S+2 G / R_{\lambda}$ approaches 0 reflect the different physical processes associated with the advection, production, diffusion and dissipation of kinetic energy and hence the different forms that Eq. (3.2) will assume in each flow. It should therefore be more meaningful to examine the dependence of $S$ on $R_{\lambda}$ in each flow rather than by indiscriminately lumping together data for $S$ from all flows, as was done in the past, e.g. Van Atta \& Antonia (1980); Sreenivasan \& Antonia (1997).

The financial support of the 'Australian Research Council' (ARC) is gratefully acknowledged. We also benefited from very fruitful discussions with Luminita Danaila during her stay in Newcastle. 


\section{REFERENCES}

Antonia, RA \& Mi, J 1993 Temperature dissipation in a turbulent round jet. J. Fluid Mech. 250 (1), 531-551.

Antonia, R. A., Anselmet, F. \& Chambers, A. J. 1986 Assessment of local isotropy using measurements in a turbulent plane jet. J. Fluid Mech. 163, 365-391.

Antonia, R. A. \& Burattini, P. 2006 Approach to the 4/5 law in homogeneous isotropic turbulence. J. Fluid Mech. 550, 175-184.

Antonia, R. A., Satyaprakash, B. R. \& Hussain, A. K. M. F. 1980 Measurements of dissipation rate and some other characteristics of turbulent plane and circular jets. Phys. Fluids 23 (4), 695-700.

Antonia, R. A., Zhou, T., Danaila, L. \& Anselmet, F. 2000 Streamwise inhomogeneity of decaying grid turbulence. Phys. Fluids 12, 3086-3089.

Baldyga, J., Bourne, J.R. \& Zimmermann, B. 1994 Investigation of mixing in jet reactors using fast, competitive, consecutive reactions. Chemical Engineering Science 49 (12), 19371946.

Batchelor, G. K. \& Townsend, A. A. 1947 Decay of vorticity in isotropic turbulence. Proc. Roy. Soc. London 190 (1023), 534-550.

Burattini, P., Antonia, R. A. \& Danaila, L. 2005 a Scale-by-scale energy budget on the axis of a turbulent round jet. J. Turbulence 6, 1-11.

Burattini, P., Antonia, R. A. \& Danaila, L. $2005 b$ Similarity in the far field of a turbulent round jet. Phys. Fluids 17, 025101.

Danaila, L., Krawczynski, J.F., Thiesset, F. \& Renou, B 2012 Yaglom-like equation in axisymmetric anisotropic context. Phys. D 241, 216223.

Darisse, A., Lemay, J. \& Benaïssa, A. 2014 Extensive study of temperature dissipation measurements on the centerline of a turbulent round jet based on the $\theta^{2} / 2$ budget. Exp . Fluids 55 (1), 1-15.

Ewing, D., Frohnapfel, B., George, W. K., Pedersen, J.M. \& Westerweel, J. 2007 Two-point similarity in the round jet. J. Fluid Mech. 577 (1), 309-330.

Friehe, C. A., VAn Atta, C. W. \& Gibson, C. H. 1972 Jet turbulence dissipation rate measurements and correlations. Turbulent shear flows, AGARD conference proceedings 93, 18.

George, W.K. 1992 The decay of homogeneous isotropic turbulence. Phys. Fluids 4, 14921509.

Kolmogorov, A. 1941 Dissipation of energy in the locally isotropic turbulence. Dokl. Akad. Nauk. SSSR 125, 15-17.

Lee, S.K., Duenidi, L., Antonia, R.A. \& Danaila, L. 2013 On the destruction coefficients for slightly heated decaying grid turbulence. Int. J. Heat and Fluid Flow 43 (0), $129-136$.

LindBorg, E. 1999 Correction to the four-fifths law due to variations of the dissipation. Phys. of Fluids 11, 510-512.

Lipari, G. \& Stansby, P. K. 2011 Review of experimental data on incompressible turbulent round jets. Flow, turbulence and combustion 87 (1), 79-114.

Lundgren, T. S. 2002 Kolmogorov two-thirds law by matched asymptotic expansion. Phys. Fluids 14, 638.

Mi, J., Xu, M. \& Zhоu, T. 2013 Reynolds number influence on statistical behaviors of turbulence in a circular free jet. Phys. Fluids 25 (7), 075101.

Panchapakesan, N. R. \& Lumley, J. L. 1993 Turbulence measurements in axisymmetric jets of air and helium. Part 1. air jet. J. Fluid Mech. 246, 197-223.

Pope, S. B. 1978 An explanation of the turbulent round-jet/plane-jet anomaly. AIAA Journal 16 (3), 279-281.

Sreenivasan, K. R. \& Antonia, R. A. 1997 The phenomenology of small-scale turbulence. Annu. Rev. Fluid Mech. 29, 435-472.

Taub, G. N., Lee, H., Balachandar, S. \& Sherif, S. A. 2013 A direct numerical simulation study of higher order statistics in a turbulent round jet. Phys. Fluids 25 (11), 115102.

Van Atta, C. W. \& Antonia, R. A. 1980 Reynolds number dependence of skewness and flatness factors of turbulent velocity derivatives. Phys. Fluids 23 (2), 252-257. 